\title{
Flexible spintronic devices on Kapton
}

Bedoya-Pinto, Amilcar; Donolato, Marco; Gobbi, Marco; Hueso, Luis E.; Vavassori, Paolo

\section{Published in:}

Applied Physics Letters

Link to article, DOI:

$10.1063 / 1.4865201$

Publication date:

2014

Document Version

Publisher's PDF, also known as Version of record

Link back to DTU Orbit

\section{Citation (APA):}

Bedoya-Pinto, A., Donolato, M., Gobbi, M., Hueso, L. E., \& Vavassori, P. (2014). Flexible spintronic devices on Kapton. Applied Physics Letters, 104(6), 062412. https://doi.org/10.1063/1.4865201

\section{General rights}

Copyright and moral rights for the publications made accessible in the public portal are retained by the authors and/or other copyright owners and it is a condition of accessing publications that users recognise and abide by the legal requirements associated with these rights.

- Users may download and print one copy of any publication from the public portal for the purpose of private study or research.

- You may not further distribute the material or use it for any profit-making activity or commercial gain

- You may freely distribute the URL identifying the publication in the public portal

If you believe that this document breaches copyright please contact us providing details, and we will remove access to the work immediately and investigate your claim. 


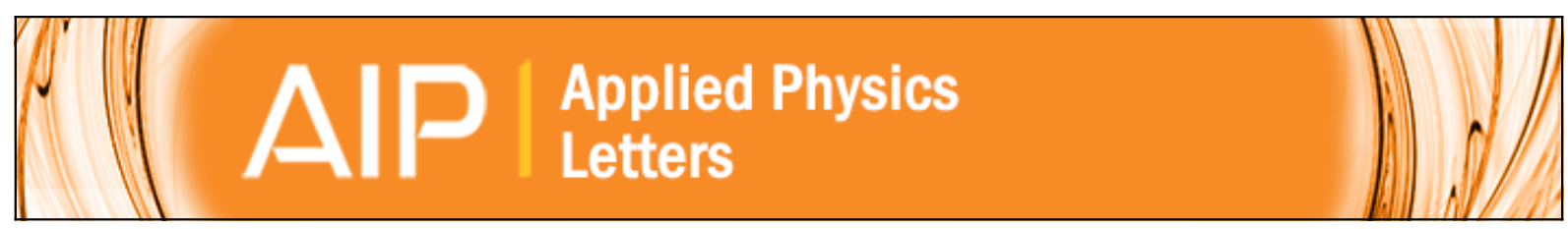

\section{Flexible spintronic devices on Kapton}

Amilcar Bedoya-Pinto, Marco Donolato, Marco Gobbi, Luis E. Hueso, and Paolo Vavassori

Citation: Applied Physics Letters 104, 062412 (2014); doi: 10.1063/1.4865201

View online: http://dx.doi.org/10.1063/1.4865201

View Table of Contents: http://scitation.aip.org/content/aip/journal/apl/104/6?ver=pdfcov

Published by the AIP Publishing

$\stackrel{A}{A} \mathbb{P} P$ Re-register for Table of Content Alerts

Create a profile.

Sign up today! 


\title{
Flexible spintronic devices on Kapton
}

\author{
Amilcar Bedoya-Pinto, ${ }^{1, \text { a) }}$ Marco Donolato, ${ }^{2}$ Marco Gobbi, ${ }^{1}$ Luis E. Hueso, ${ }^{1,3}$ \\ and Paolo Vavassori ${ }^{1,3, b)}$ \\ ${ }^{1}$ CIC nanoGUNE Consolider, 20018 Donostia-San Sebastian, Spain \\ ${ }^{2}$ DTU Nanotech, Building 345 East, DK-2800 Kongens Lyngby, Denmark \\ ${ }^{3}$ IKERBASQUE, Basque Foundation for Science, 48011 Bilbao, Spain
}

(Received 8 October 2013; accepted 20 January 2014; published online 12 February 2014; publisher error corrected 19 February 2014)

\begin{abstract}
Magnetic tunnel junctions and nano-sized domain-wall conduits have been fabricated on the flexible substrate Kapton. Despite the delicate nature of tunneling barriers and zig-zag shaped nanowires, the devices show an outstanding integrity and robustness upon mechanical bending. High values of bending angle $(\mathrm{r}=5 \mathrm{~mm})$ have been achieved without degradation of the device performance, reaching room-temperature tunneling magnetoresistance ratios of $12 \%$ in bended $\mathrm{Co} / \mathrm{Al}_{2} \mathrm{O}_{3} / \mathrm{NiFe}$ junctions. In addition, a suitable route to pattern high-quality nanostructures directly on the polyimide surface is established. These results demonstrate that Kapton is a promising platform for low-cost, flexible spintronic applications involving tunnel junction elements and nanostructurization. (C) 2014 AIP Publishing LLC. [http://dx.doi.org/10.1063/1.4865201]
\end{abstract}

The integration of different classes of micro and nanodevices on non-conventional substrates is receiving considerable interests due to the continuous growth of the flexible electronics market. For what concerns magneto-electronic and spintronic devices, while the deposition of magnetic multilayer structures on flexible substrates has been shown since the last decade, ${ }^{1}$ the first fully functional magnetic devices on non-planar substrates have been just recently achieved. ${ }^{2}$ The possibility of exploiting additional materials properties and the need to address a variety of application areas have led to innovative approaches, as the realization of stretchable ${ }^{2,3}$ or printable ${ }^{4,5}$ giant magneto-resistance (GMR) based sensors, which, as main feature, show isotropic field sensitivity. ${ }^{6}$ Moreover, advances in strain engineering ${ }^{7}$ have allowed the integration of such GMR sensors even in rolled-up architectures. ${ }^{8-10}$

While the progress and development of GMR-based devices on non-planar substrates have been remarkable, few groups have been working on the realization of magnetic tunnel junctions (MTJs) on flexible substrates so far. Growing mechanically robust and defect-free tunneling barriers over flexible and non-planar substrates is a crucial technological challenge towards the integration of nonvolatile magnetic random access memories (MRAMs) and magnetic field sensors over advanced non-planar architectures. Recently, it has been demonstrated that magnetic tunnel junctions with a $\mathrm{MgO}$ barrier can be grown on top of surface-treated polyester-based organic substrates, ${ }^{11}$ while some other works address the possibility of integrating tunnel junction elements into bendable membranes. ${ }^{12}$ In this context, organic spintronic devices such as spin-valves ${ }^{13,14}$ or magnetic tunnel transistors ${ }^{15}$ would also benefit from the integration on polymeric templates, to envision flexible, all-organic architectures.

Besides thin film deposition, patterning magnetic devices on non-conventional substrates is an extremely delicate

\footnotetext{
${ }^{\text {a)} E l e c t r o n i c ~ m a i l: ~ a . b e d o y a @ n a n o g u n e . e u ~}$

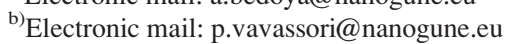

process, since heating, chemical or physical etching processes can affect or damage the substrate as well as the magnetic thin film. Remarkably, the possibility of patterning or even transferring magnetic nanostructures - based on the control of geometrically constrained magnetic domain walls-into elastomeric substrates, has been recently demonstrated. ${ }^{12}$ However, structuring devices of lateral sizes down to few hundred nanometers using standard techniques directly onto a non-planar substrate has not been achieved so far.

Among non-planar templates for flexible electronic applications, polymeric substrates, such as aromatic polyimides, are attractive candidates due to their excellent thermal stability and good mechanical, optical, electrical, and chemical properties. ${ }^{16}$ While they are currently widely used for flexible and high-density electronic interconnection applications, their integration with magnetic and spintronic devices is still lacking.

In this paper, we demonstrate that Kapton, a polyimide, is a very promising flexible substrate for magneto-electronic applications. For this purpose, we characterize $\mathrm{Al}_{2} \mathrm{O}_{3}$-based MTJs as well as magnetic nanowires grown and patterned directly on a Kapton substrate. As a first step, we study the variation of the magnetic properties of a Cobalt (Co) and Permalloy $\left(\mathrm{Ni}_{81} \mathrm{Fe}_{19}\right.$, hereafter named $\left.\mathrm{NiFe}\right)$ thin film deposited on Kapton with respect to a rigid Silicon/ $/ \mathrm{SiO}_{2}$ substrate. Furthermore, we analyze the magnetoresistive response and robustness of large-area $\mathrm{Co} / \mathrm{Al}_{2} \mathrm{O}_{3} / \mathrm{NiFe}$ magnetic tunnel junctions as a function of the bending angle of the Kapton substrate. Finally, we show that patterned nanosized magnetic devices (NiFe domain-wall conduits) previously used for nanoscale magnetic beads transportation ${ }^{17}$ can be defined on the same substrate using electron beam lithography, and are found to show unchanged magnetoresistive properties after bending. The outstanding integrity of these devices upon mechanical stress, reflected in record values of bending angle without degradation of the device performance, demonstrates that Kapton is a promising platform to host sophisticated devices for flexible spintronic applications. 
Kapton substrates have been purchased from DuPont in form of individual sheets as well as adhesive tape. Before magnetic thin film deposition, the substrates have been carefully cleaned in ultrasound using both acetone and isopropanol. NiFe and Co films $(t=10 \mathrm{~nm})$ have been deposited by means of electron-beam evaporation in an ultra high vacuum chamber ( $\mathrm{p}_{\text {base }}=5 \times 10^{-11}$ millibars). The $\mathrm{Al}_{2} \mathrm{O}_{3}$ barrier of the magnetic tunnel junctions was produced by deposition of a thin aluminum layer $(t=2 \mathrm{~nm})$ and subsequent $\mathrm{O}_{2}$-plasma oxidation. The cross-geometry of the junction has been defined via in-situ shadow masking. The junction area amounts to $250 \times 250 \mu \mathrm{m}^{2}$. The crystallographic grain size and surface roughness of the metal layers have been determined by means of a commercial Philips X-pert diffractometer (XRD) and an Agilent 5500 atomic force microscope (AFM), respectively. An Evico ${ }^{\circledR}$ magneto-optical Kerr effect (MOKE) microscope ${ }^{18}$ has been used for the magnetic characterization. The hysteresis loops have been recorded over a metal film with a total area of $5 \mathrm{~mm} \times 5 \mathrm{~mm}$. The magnetic field has been applied in the film plane and swept between \pm 500 Oe. Magneto-transport measurements have been performed in a commercial 4-probe Lake Shore Probe Station with an in-plane magnetic field ( $\pm 200 \mathrm{Oe})$. For the bending experiments, the samples have been fixed along semi-circular metallic blocks with different curvature radii.

Prior to device fabrication, the effect of the Kapton substrate on the magnetic properties of the studied metal thin films has been investigated. Figures 1(a) and 1(b) compare the magnetic properties of $15 \mathrm{~nm}$-thick films (Co, NiFe) grown on $\mathrm{Si} / \mathrm{SiO}_{2}$ and Kapton, respectively. For both ferromagnetic metals, the width of the hysteresis loop increases when the film is grown on Kapton. The increase is remarkable for the Co case, which displays a coercive field more than five times larger. The same effect has been observed both on films patterned on a large area and on a stripe of $300 \mu \mathrm{m}$ width. On the other hand, AFM images (Figures 1(e) and 1(f)) suggest that the surface morphology is barely affected when the metals are grown on Kapton with respect to the $\mathrm{Si} / \mathrm{SiO}_{2}$ reference. The surface roughness, as quantified by the root-mean square (RMS) average, amounts to $0.3 \mathrm{~nm}(0.4 \mathrm{~nm})$ for the $\mathrm{Si} / \mathrm{SiO}_{2}($ Kapton$)$ substrate, respectively. These observations indicate that the remarkable increase of the coercive field of the metals grown on Kapton cannot be attributed to a change in the magnetization reversal process due to a more disordered growth, which should be clearly reflected on the surface topography and average surface roughness. Another possibility to explain the modulation of the coercive field is a substrate-induced change in the crystalline properties of the ferromagnet. As expected from the substrates used for growth $\left(\mathrm{Si} /\right.$ amorphous $\mathrm{SiO}_{2}$ and Kapton), the XRD characterization confirms that both Co and NiFe metal layers are polycrystalline. In such cases, the coercivity is found to have a strong dependence on the grain size. ${ }^{19}$ Figures 1 (c) and 1(d) show the $\theta-2 \theta$ scan of Co and $\mathrm{NiFe}$ layers on $\mathrm{SiO}_{2} /$ Kapton around the (111) reflection, where the grain size analysis has been performed. Using the Scherrer-Formula $^{20}$ for spherical grains of diameter $d$, the results yield a smaller grain size for Co $(d=5.8 \mathrm{~nm})$ and $\mathrm{NiFe}(d=6.6 \mathrm{~nm})$ grown on Kapton with respect to Co $(d=7.6 \mathrm{~nm})$ and $\mathrm{NiFe}(d=8.1 \mathrm{~nm})$ layers on the $\mathrm{Si} / \mathrm{SiO}_{2}$ substrate. A comparable decrease of the grain size (less than a factor of two) leading to a remarkable increase in coercivity (x5) has been previously observed by Bae $e t$ al. ${ }^{21}$ in $\mathrm{NiFe}$ and $\mathrm{CoFe}$ layers, and was attributed to differences in nucleation and surface kinetics triggered by the fabrication process. In our case, as there are no differences in the fabrication (a)

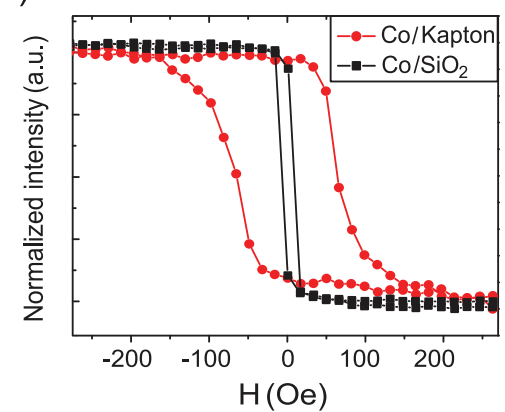

(b)

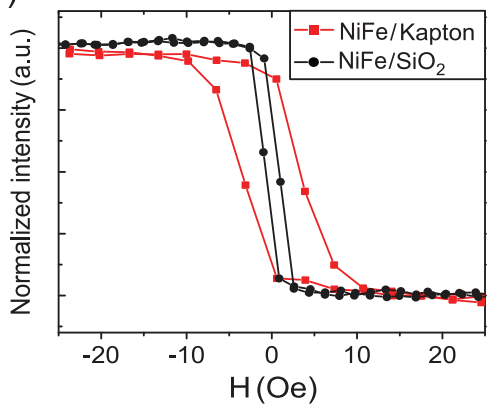

(c)

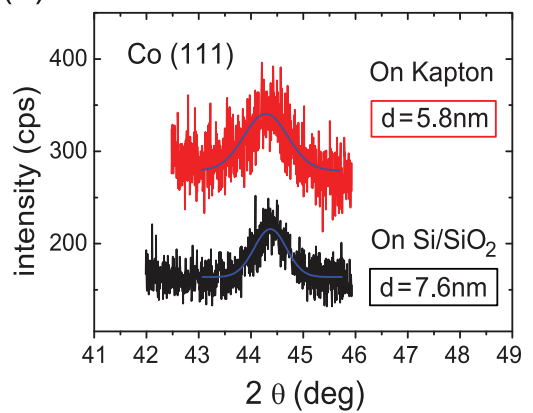

(d)

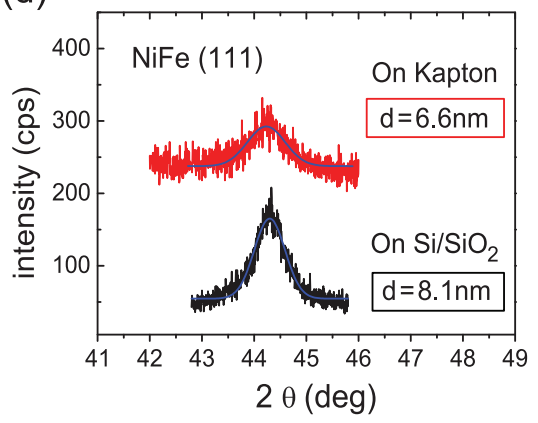

(e)

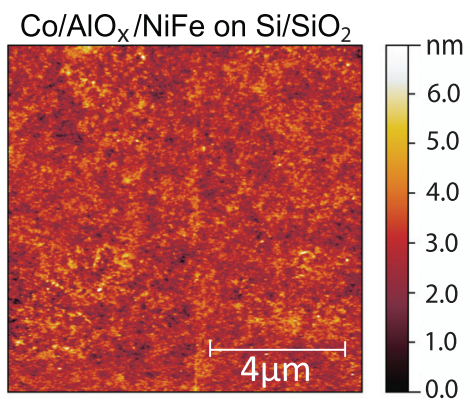

(f)

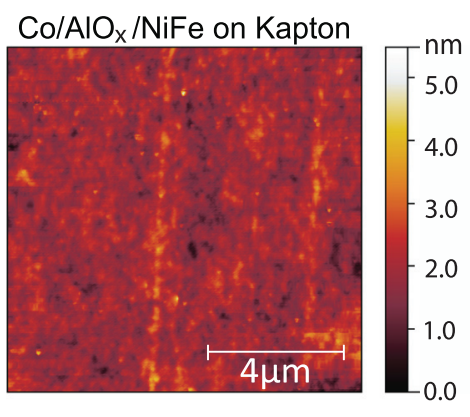

FIG. 1. Magneto-Optical Kerr signal of $\mathrm{Co}$ (a) and $\mathrm{NiFe}$ (b) thin films deposited on $\mathrm{SiO}_{2} /$ Kapton substrates, showing a clear difference of the coercive fields in either case. X-ray diffraction patterns of the $\mathrm{Co}(111)$ (c) and $\mathrm{NiFe}(111)$ (d) reflections of the polycrystalline metallic layers grown on $\mathrm{SiO}_{2} / \mathrm{Kapton}_{\text {substrates. }}$ The derived crystallographic grain sizes are given for comparison. AFM images of the junction area over a device on $\mathrm{Si} / \mathrm{SiO}{ }_{2}$ (e) and $\mathrm{Kapton}$ (f), showing that the surface topography of the junctions is very similar in the two cases. 
strategy (e.g., growth rate, deposition pressure, substrate temperature), it seems that the Kapton surface itself induces a different nucleation process, affecting the magnetic reversal but preserving a good quality of the magnetic films. Following the argument put forward in Ref. 19, the coercive field enhancement as the material grain size decreases is due to a transition from disordered, incoherent, magnetization reversal (larger grain size) to a progressively more coherent reversal process (smaller grain size). This transition, and thus the change in the coercive field, is expected to be more relevant when the grain size is varied across or near the material exchange length, as in our case (the exchange length in $\mathrm{NiFe}$ and $\mathrm{Co}$ is $\sim 5 \mathrm{~nm}$ ).

The ability to modify the magnetic properties while preserving ideal growth morphology is a key feature that allows the fabrication of sensitive devices. For instance, the performance of magnetic tunnel junctions is extremely sensitive to the quality of the insulating barrier. Rough barriers might lead to electrical shortcuts and to magnetic pinning effects of the ferromagnetic layers, which might induce local fields at the ferromagnet/insulator interface which are not desired for an efficient spin injection and tunneling. In our case, as the magnetic thin films grow very smooth on Kapton, there is no technical impediment to achieve a working magnetic tunnel junction. In fact, the $\mathrm{Co} / \mathrm{Al}_{2} \mathrm{O}_{3} / \mathrm{NiFe}$ devices on Kapton show even a larger tunneling magnetoresistance ratio (TMR) than the ones on $\mathrm{Si} / \mathrm{SiO}_{2}$ grown in the same run, as shown in Figure 2(b). The remarkable increase in the coercive field in the Co-electrode on Kapton (see Figure 1(a)) has a substantial impact on the tunneling magnetoresistance, as it allows a more defined antiparallel state, leading to TMR values of $12 \%$ at room temperature. Moreover, the robustness of the MTJs on Kapton upon bending has been tested by placing the device in semicircular metallic blocks with different radii $r$. A photograph of the experiment is presented in Figure 2(a).

The magneto-transport measurements as a function of bending angle, summarized in Figure 2(b), show a negligible difference in the TMR ratio of the bended devices with respect to the planar ones, suggesting that particularly the $\mathrm{Al}_{2} \mathrm{O}_{3}$ tunnel barrier is able to stand a high yield of mechanical stress without cracks or electrical shortcuts. It is worth to mention that each of the measurements has been done in the bended state, which means that the sample has been exposed to mechanical stress during a considerable amount of time, unlike previous studies which measure flexible devices in a planar geometry before- and after short-time-bending. ${ }^{11}$ In addition, the achievement of tensile strain corresponding to a bending radius of $r=10 \mathrm{~mm}$ without degradation of the MTJ performance is the highest reported to date. Note that an unaffected TMR response has been even achieved up to a bending radius of $\mathrm{r}=5 \mathrm{~mm}$ in a device with a nominally thicker barrier $(\mathrm{t}=2.5 \mathrm{~nm})$ and thus lower magnetoresistance (see Figure S1 in Supplementary Information ${ }^{22}$ ). The fact that the TMR is substantially independent of the bending, despite the high magnetostriction coefficient of $\mathrm{Co}$, means that very little strain is transferred from the substrate to the MTJ structure upon bending. The strain induced at the Kapton/Co interface is derived from the bending radius and geometrical parameters. ${ }^{22}$ All these unique properties place the (a)

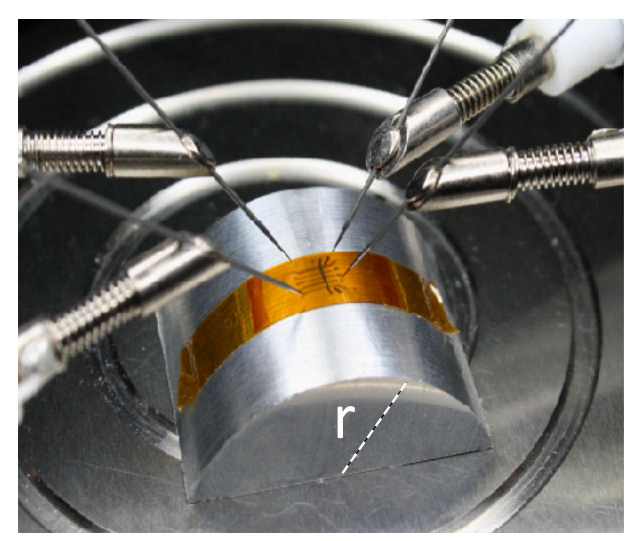

(b)

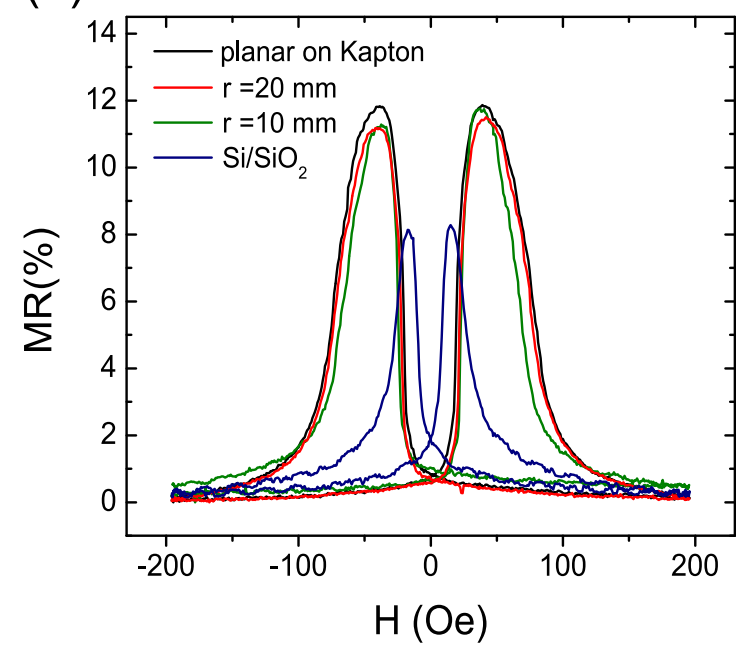

FIG. 2. (a) Photograph of the bending angle magnetotransport experiment; the radius of the curved support is used as a parameter in the device characterization. (b) TMR ratio of a $\mathrm{Co} / \mathrm{Al}_{2} \mathrm{O}_{3} / \mathrm{NiFe}$ device grown on $\mathrm{Si} / \mathrm{SiO}_{2}$ and on Kapton, in the latter case as a function of bending angle.

polyimide-based substrate Kapton as a promising platform for the production of flexible spin tunneling devices such as MRAMs.

The next aspect to consider is the suitability of the substrate to support nanostructurization. Magnetic nanowires, in the form of zig-zag shaped domain-wall conduits, ${ }^{23}$ are a particularly demanding test for checking the preservation of their integrity and magnetic functionality. Their magnetization reversal occurs via propagation of domain walls along the wire length, a process which relies crucially on the full integrity of the magnetic nanowires. This is the reason why they are chosen as an illustrative example.

The nanowires have been patterned on the Kapton substrate via electron-beam lithography. A thin layer of gold $(1.5 \mathrm{~nm})$ is evaporated on top of the polymethilmethacrilate (PMMA) electron beam sensitive layers in order to achieve substrate conductivity. After e-beam exposure and before developing the PMMA layer, the gold layer is removed by means of a commercial Au-etchant (Sigma-Aldrich). Finally, a $30 \mathrm{~nm}$-thick NiFe layer is deposited via electron-beam evaporation and the residual PMMA is lifted off. Figure 3(a) shows the lithography process workflow. Following this procedure, nanostructures having size as low as $100 \mathrm{~nm}$ can be 
(a)
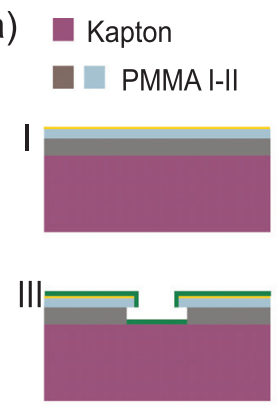

IV

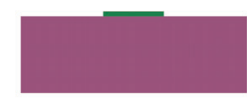

(c)

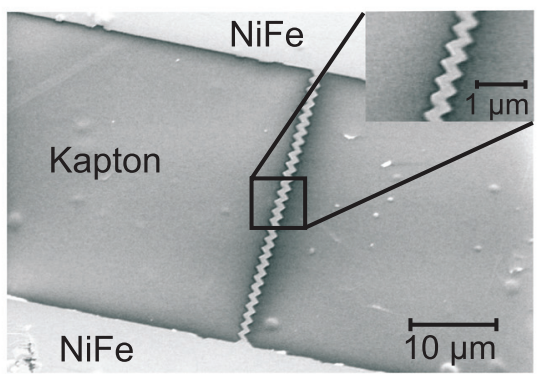

(b)

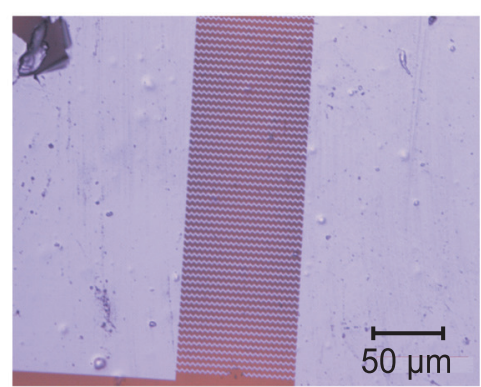

(d)

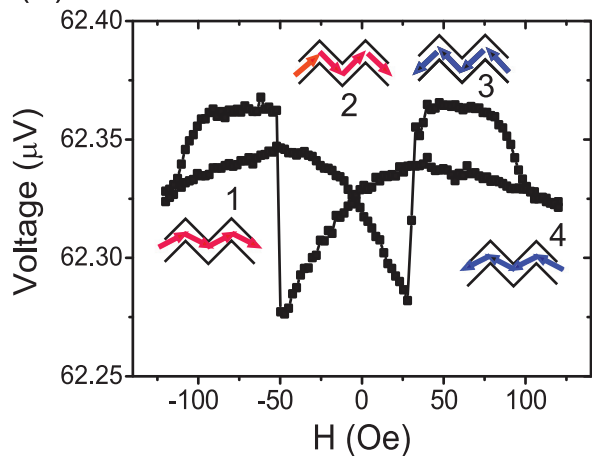

FIG. 3. (a) Scheme of the fabrication process of the magnetic nanowires on the Kapton substrate. (b) Optical microscope image of an array of zigzag domainwall conduits patterned on the Kapton substrate. (c) Electron beam microscopy image of a single domain-wall conduit imaged when bended over a radius of $\mathrm{r}=10 \mathrm{~mm}$, showing the full integrity of the wire. (d) Magneto-transport measurement of the same single magnetic nanowire in the bended state ( $\mathrm{r}=10 \mathrm{~mm}$ ). The field is swept on the nanowire plane, perpendicular to the zigzag direction.

attained on the Kapton substrate without damage or substantial loss of precision with respect to the use of Si substrate.

An array of zigzag wires having a width of $\sim 500 \mathrm{~nm}$ fabricated on a Kapton substrate is shown in Figure 3(b). The structures are generally preserved after the lithographic steps previously described. Figure 3(c) shows a scanning electron microscope image of an individual $\mathrm{NiFe}$ wire $(t=300 \mathrm{~nm})$, bended with the procedure described in Figure (a) to a radius of $r=10 \mathrm{~mm}$. These individual nanowires are subjected to anisotropic magnetoresistance (AMR) measurements, in order to test their magnetic integrity upon bending. The results are shown in Figure 3(d), including the schematics of the magnetization reversal at each regime. The magnetic field is applied parallel to the zigzag wire and swept between \pm 120 Oe. At high fields, the magnetic domains are aligned to the field, then, by reducing the external field magnitude the shape anisotropy acts to force the magnetization along the zig-zag side direction, thus causing a decrease in the voltage. When the external field direction is reversed, the switch of the magnetization direction in the wire causes the measured step in the voltage signal. ${ }^{24}$ The slight asymmetry between the two branches of the AMR curves it is most likely due to an incomplete magnetic saturation of the zig-zag wire. As discussed in Ref. 25, in magnetic nanostructured devices the incomplete erasure of magnetic metastable configurations nucleated in magnetization reversal during the initial branch of the AMR curve results in a slightly different return branch and thus to the appearance of asymmetries in the AMR response.

As a technological outlook, the robustness of the magnetic nanowires upon bending and the ability of patterning conductive magnetic nanostructures on a polymeric substrate are of great interest both towards the realization of flexible recording media ${ }^{26}$ and the integration of magnetic nanodevices into polymeric microfluidic platforms. ${ }^{12}$

In summary, we have fabricated high-quality devices such as magnetic tunnel junctions and zig-zag-shaped domain-wall conduits on the flexible substrate Kapton. While the structural quality of the magnetic layers is preserved, the Kapton substrate does have a sizable effect on the magnetic properties of the layers, which are reflected in a higher magnetoresistance ratio (12\%) of the magnetic tunnel junctions grown on Kapton as compared to the ones grown on the rigid Si-substrate. Most importantly, the magnetic tunnel junction performance is not affected upon substrate bending up to a radius of $r=5 \mathrm{~mm}$, demonstrating the potential of these devices for flexible spintronic applications. As a technological progress, nano-sized arrays have been patterned via electronbeam lithography directly on the Kapton surface with comparable quality and resolution as in the conventional rigid substrates. In this way, zig-zag shaped magnetic nanowires have been defined and characterized, showing robust magnetotransport properties which, despite of the reduced dimensions, do not degrade upon substrate bending. These results demonstrate that low-cost, flexible polyimide substrates such as Kapton are fully compatible with the integration of spintronic devices having delicate interface layer properties-such as MTJs - and with advanced fabrication techniques such as electron beam lithography. These achievements are of great technological interest and can pave the way to the development of multiple functionalities in flexible spintronic devices.

The authors acknowledge R. Llopis and C. Rufo for technical support. This work was supported by the European 
Union 7th Framework Programme (NMP3-SL-2011-263104HINTS), by the European Research Council (Grant No. 257654-SPINTROS), by the Basque Government under Project Nos. PI2011-1 and PI2012-47, and by the Spanish Ministry of Economy and Competitiveness under Project Nos. MAT2012-36844 and MAT2012-37638. M.D. acknowledges the Ørsted Research Grant.

${ }^{1}$ S. S. P. Parkin, Appl. Phys. Lett. 69, 3092 (1996).

${ }^{2}$ M. Melzer, D. Makarov, A. Calvimontes, D. Karnaushenko, S. Baunack, R. Kaltofen, Y. Mei, and O. G. Schmidt, Nano Lett. 11, 2522 (2011).

${ }^{3}$ M. Melzer, G. Lin, D. Makarov, and O. G. Schmidt, Adv. Mater. 24, 6468 (2012).

${ }^{4}$ D. Karnaushenko, D. Makarov, C. Yan, R. Streubel, and O. G. Schmidt, Adv. Mater. 24, 4518 (2012).

${ }^{5}$ D. Makarov, D. Karnaushenko, and O. G. Schmidt, ChemPhysChem 14, 1771 (2013).

${ }^{6}$ M. Melzer, D. Karnaushenko, D. Makarov, L. Baraban, A. Calvimontes, I. Mönch, R. Kaltofen, Y. Mei, and O. G. Schmidt, RSC Adv. 2, 2284 (2012).

${ }^{7}$ O. G. Schmidt and K. Eberl, Nature 410, 168 (2001).

${ }^{8}$ I. Mönch, D. Makarov, R. Koseva, L. Baraban, D. Karnaushenko, C. Kaiser, K.-F. Arndt, and O. G. Schmidt, ACS Nano 5, 7436 (2011).

${ }^{9}$ C. Müller, C. C. B. Bufon, D. Makarov, L. E. Fernandez-Outon, W. A. A. Macedo, O. G. Schmidt, and D. H. Mosca, Nanoscale 4, 7155 (2012).

${ }^{10}$ R. Streubel, D. J. Thurmer, D. Makarov, F. Kronast, T. Kosub, V. Kravchuk, D. D. Sheka, Y. Gaididei, R. Schäfer, and O. G. Schmidt, Nano Lett. 12, 3961 (2012).
${ }^{11}$ C. Barraud, C. Deranlot, P. Seneor, R. Mattana, B. Dlubak, S. Fusil, K. Bouzehouane, D. Deneuve, F. Petroff, and A. Fert, Appl. Phys. Lett. 96, 072502 (2010).

${ }^{12}$ M. Donolato, C. Tollan, J. M. Porro, A. Berger, and P. Vavassori, Adv. Mater. 25, 623 (2013).

${ }^{13}$ B. Li, C.-Y. Kao, J.-W. Yoo, V. N. Prigodin, and A. J. Epstein, Adv. Mater. 23, 3382 (2011).

${ }^{14}$ M. Gobbi, F. Golmar, R. Llopis, F. Casanova, and L. E. Hueso, Adv. Mater. 23, 1609 (2011).

${ }^{15}$ M. Gobbi, A. Bedoya-Pinto, F. Golmar, R. Llopis, F. Casanova, and L. E. Hueso, Appl. Phys. Lett. 101, 102404 (2012).

${ }^{16}$ Y. Kim, J. Keum, J. Lee, H. Lim, and C. Ha, Adv. Mater. Opt. Electron. 10, 273 (2000).

${ }^{17}$ M. Donolato, P. Vavassori, M. Gobbi, M. Deryabina, M. F. Hansen, V. Metlushko, B. Ilic, M. Cantoni, D. Petti, S. Brivio, and R. Bertacco, Adv. Mater. 22, 2706 (2010).

${ }^{18}$ E. Nikulina, O. Idigoras, P. Vavassori, A. Chuvilin, and A. Berger, Appl. Phys. Lett. 100, 142401 (2012).

${ }^{19}$ G. Herzer, IEEE Trans. Magn. 26, 1397 (1990).

${ }^{20}$ A. L. Patterson, Phys. Rev. 56, 978 (1939).

${ }^{21}$ S. Bae, J. Li, J. H. Judy, and S. Zurn, Appl. Phys. Lett. 77, 3435 (2000).

${ }^{22}$ See supplementary material at http://dx.doi.org/10.1063/1.4865201 for TMR measurements up to a bending radius of $\mathrm{r}=5 \mathrm{~mm}$ and the estimation of the induced strain at the Kapton/Co interface.

${ }^{23}$ M. Donolato, F. Lofink, S. Hankemeier, J. M. Porro, H. P. Oepen, and P. Vavassori, J. Appl. Phys. 111, $07 B 336$ (2012).

${ }^{24}$ F. C. S. da Silva, W. C. Uhlig, A. B. Kos, S. Schima, J. Aumentado, J. Unguris, and D. P. Pappas, Appl. Phys. Lett. 85, 6022 (2004).

${ }^{25}$ P. Vavassori, M. Grimsditch, V. Metlushko, N. Zaluzec, and B. Ilic, Appl. Phys. Lett. 86, 072507 (2005).

${ }^{26}$ S. Kim, H. Y. Jeong, S. K. Kim, S.-Y. Choi, and K. J. Lee, Nano Lett. 11, 5438 (2011). 